\title{
Moderate Exercise Restores Pancreatic Beta-Cell Function and Autonomic Nervous System Activity in Obese Rats Induced by High-Fat Diet
}

\author{
Rodrigo Mello Gomes ${ }^{a}$ Laize Peron Tófolo ${ }^{a}$ Wilson Rinaldib Dionizia Xavier \\ Scomparina Sabrina Grassiollia Luiz Felipe Barella ${ }^{a}$ Júlio Cezar de Oliveira ${ }^{a}$ \\ Renato Chaves Souto Branco ${ }^{a}$ Aryane Rodrigues Agostinho ${ }^{a}$ Tatiane Aparecida da \\ Silva Ribeiro ${ }^{b}$ Clarice Gravena ${ }^{a}$ Paulo Cezar de Freitas Mathias ${ }^{a}$ \\ aDepartment of Cell Biology and Genetic, State University of Maringá, Maringá PR Brazil; bepartment \\ of Physical Education, State University of Maringá, Maringá PR Brazil
}

\section{Key Words}

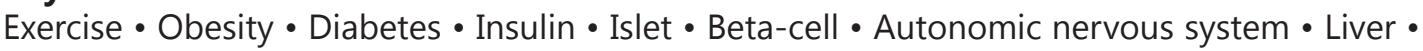
High-fat diet

\begin{abstract}
Background/Aims: Metabolic syndrome has been identified as one of the most significant threats to human health in the $21^{\text {st }}$ century. Exercise training has been shown to counteract obesity and metabolic syndrome. The present study aimed to investigate the effects of moderate exercise training on pancreatic beta-cell function and autonomic nervous system (ANS) activity in rats fed a high-fat diet (HFD). Methods: Weaning rats were divided into four groups: rats fed a standard chow or HFD (sedentary, Control-SED and HFD-SED; or exercised, Control-EXE and HFD-EXE, respectively). Exercised rats ran (from 21- to 91-daysold) for 60 minutes ( 3 times/week) over a 10-week period. Glucose and insulin tolerance tests were performed. Pancreatic islets were isolated to study glucose-induced insulin secretion (GIIS). Parasympathetic and sympathetic nerve electrical signals were measured, and liver samples were processed and histologically analyzed. Results: Exercise prevented obesity, insulin resistance, and liver steatosis as well as improved total cholesterol, ALT, and AST levels. Islets from HFD rats showed insulin hypersecretion which was ameliorated by exercise. Exercise decreased vagal nerve activity in the HFD-EXE group and increased the activity of the sympathetic nervous system in both exercised groups. Conclusion: Exercise prevents obesity and liver steatosis and restores pancreatic beta-cell function and ANS activity in HFD-obese rats.




\section{Introduction}

Contemporary lifestyle is often characterized by physical inactivity and consumption of a high-fat diet (HFD). These factors are major contributors to obesity, type 2 diabetes, disorders in the activity of the autonomic nervous system (ANS) and liver steatosis [1,2].

In general, obese individuals are insulin resistant, hyperinsulinemic and their pancreatic islets secrete more insulin compared to the pancreatic islets of lean individuals. Likewise, obese subjects have an imbalance in ANS activity, which is characterized by an increase in parasympathetic nervous system (PNS) tonus and/or a decrease in the sympathetic nervous system (SNS) tonus. These imbalances in ANS activity indicate an important relationship with the regulation of insulin secretion [3]. Furthermore, epidemiological studies show a strong association between obesity onset and the ingestion of high calorie diets and physical inactivity. However, physical activity may prevent the development of obesity and metabolic syndrome [4,5]. Scomparin et al. showed that hyperinsulinemia, insulin resistance and imbalanced ANS activity were inhibited in monosodium L-glutamate (MSG) obese mice subjected to early swim exercise training [6]. In addition to inhibiting the onset of obesity, moderate physical exercise also results in corrections in insulin tissue sensitivity and glucose-induced insulin secretion (GIIS) [7].

Several studies have shown that animals fed a HFD developed obesity, manifested a significant resistance to insulin and had insulin secretion disorders [8-10]. Ropelle et al. showed that HFD-obese rats demonstrated insulin resistance in the liver and skeletal muscle, which was accompanied by decreases in the insulin receptor substrate 1 (IRS-1), PI3K and Akt, all of which are involved in the insulin signaling pathway [11]. HFD consumption causes liver steatosis and elevation of the plasma liver enzymes, alanine aminotransferase (ALT) and aspartate aminotransferase (AST) [12]. There is little data showing the effects of alteration in ANS activity associated with obesity onset in HFD-fed rats or the effect of moderate exercise on HFD-fed rats. Furthermore, exercise is able to restore insulin sensitivity and secretion. In the present study, we evaluated the effect of moderate exercise on obesity onset, insulin resistance, insulin secretion in isolated pancreatic islets, ANS activity and liver morphology in lean and HFD-treated rats.

\section{Material and Methods}

\section{Ethical approval}

All experiments were undertaken according to norms established by the Brazilian Association for Animal Experimentation (COBEA) and previously approved by the Ethics Committee in Animal Research of the State University of Maringá (protocol number 084/2009).

\section{Animals and diet}

Twenty-one-day-old (recently weaned) male Wistar rats (Rattus norvegicus) were randomly divided into four groups: control sedentary (Control-SED), HFD sedentary (HFD-SED), control exercised (ControlEXE) and HFD exercised (HFD-EXE). All animals were kept in an environmentally controlled room (22 2 ${ }^{\circ} \mathrm{C}$ and $12 \mathrm{~h}$ light/dark cycle; lights on from 7:00 AM to 7:00 PM). Control animals received a standard chow diet (Nuvital CR1; Nuvilab ${ }^{\circledR}$, Colombo, Paraná, Brazil) with 4.3 kcal/g ad libitum. HFD-fed animals received a previously described hypercaloric diet (5.9 kcal/g) rich in saturated fat [13]. A standard of three animals per cage for all groups was set. Diet consumption and body weight were evaluated weekly after weaning (21 days old) until 91 days-old. Food intake was calculated by estimating the total area under the food intake curve (AUC), using the trapezoidal method, during the entire 10-week treatment period, from 21 until 91 days-old.

\section{Moderate treadmill exercise protocol}

Rats were trained on an animal treadmill (model ET-2000 Insight; Ribeirão Preto, São Paulo, Brazil) three times a week for a total of 10 weeks ( 30 sessions from 21 until 91 days old). Adjustments in speed and 
Gomes et al.: Moderate Exercise Restores Beta-Cell Function

Fig. 1. Moderate treadmill exercise protocol. Rats were trained on an animal treadmill three times a week for a total of 30 sessions (10 weeks). Adjustments in speed (10 up to $20 \mathrm{~m} \times \mathrm{min}^{-1}$; dashed line) and time (10 up to $60 \mathrm{~min}$; continuous line) were undertaken throughout the training program.

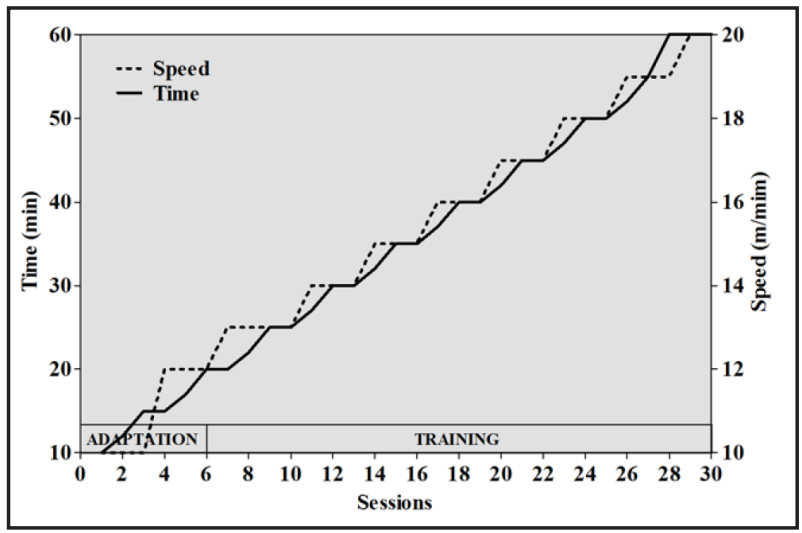

time were undertaken throughout the training program. During the first two weeks, the adaptation period, speed ranged between 10 and $12 \mathrm{~m} \times \mathrm{min}^{-1}$, and each session lasted between 10 and $15 \mathrm{~min}$. Speed was gradually increased during the main 8 -week training program from $13 \mathrm{~m} \times \mathrm{min}^{-1}$ during the first week up to $20 \mathrm{~m} \times \mathrm{min}^{-1}$ during the last week. Each session in the first week of training lasted $20 \mathrm{~min}$. Training session time increased up to $60 \mathrm{~min}$ during the sessions in the last week of training (Fig. 1). Initial and resting exercises were performed at the start and end of each training session (speed $=10 \mathrm{~m} \times \mathrm{min}^{-1}$ and duration $=5 \mathrm{~min}$ ). Treadmill apparatus inclination was not changed during the training period. Although we did not measure exercise intensity, this training protocol is a low-intensity exercise training $\left(55 \%\right.$ of $\mathrm{VO}_{2 \max } ; 16-20$ $\mathrm{m} \times \mathrm{min}^{-1}$ ) according to a previous study [14]. All experimental procedures were performed 48 hours after the last training session.

\section{Assessment of obesity}

Adult animals from each experimental group (91 days old; $\mathrm{n}=10$ - 12 per group) were anaesthetized (thiopental $45 \mathrm{mg} / \mathrm{kg}$ of body weight) and weighed, and the body length was measured to calculate the Lee index $\left(\right.$ Lee index $=\left(\right.$ body weight $[\mathrm{g}]^{1 / 3} /$ body length $\left.\left.[\mathrm{cm}]\right) \times 1000\right)$ [15]. Animals were sacrificed by decapitation so that the periepididymal (EPI), retroperitoneal (RP), inguinal (ING) and mesenteric (MES) fat pads could be removed and weighed.

\section{Intravenous glucose tolerance test (ivGTT)}

Animals from the four experimental groups (91 days old; $n=10-12$ ) were fasted for $12 \mathrm{~h}$ (from $7 \mathrm{PM}$ to $7 \mathrm{AM}$ ) before the test. Following fasting, all animals received a glucose infusion $(1 \mathrm{~g} / \mathrm{Kg}$ of body weight), and blood samples were collected by a cannula implanted in the right jugular vein at 0 min (prior to glucose infusion), 5, 15, 30 and 45 min after the infusion. Blood was centrifuged, and the plasma was used to determine the glycemia by the glucose-oxidase technique (Gold Analisa ${ }^{\circledR}$ Belo Horizonte, Minas Gerais, Brazil) and insulinemia by radio-immunoassay (RIA) (PerkinElmer ${ }^{\circledR}$, Boston, Massachusetts, USA). The glucose and insulin responses during the glucose tolerance test were calculated by estimating the total area under the glucose curve using the trapezoidal method [16]. The homeostasis model assessment of insulin resistance (HOMA) was used as the physiological index of insulin resistance. This was assessed from fasting glucose and fasting insulin concentrations using the following formula: HOMA = (fasting insulin [ng/ $\mathrm{mL}] \times$ fasting glucose $[\mathrm{mg} / \mathrm{dL}]) / 22.5$ [17]

\section{Intraperitoneal insulin tolerance test (ipITT)}

Another batch of animals from the four experimental groups (91 days old; $n=10$ - 12) received an intraperitoneal injection of human recombinant insulin $(1 \mathrm{U} / \mathrm{kg}$ of body weight; in $200 \mu \mathrm{L}$ of saline; Eli Lilly ${ }^{\circledR}$, São Paulo, Brazil). Blood samples were obtained by tail venesection, and glycemia was measured using a glucometer (ACCU-CHEK ${ }^{\circledR}$ Advantage, Roche Diagnostics, Mannheim, Germany) at 0 (prior to insulin injection), 5, 15, 30 and 45 min after injection; glycemia was measured at each correspondent time. Thereafter, the constant rate for glucose disappearance $\left(\mathrm{K}_{\mathrm{itt}}\right)$ was calculated by the formula 0.693 / $\left(t_{1 / 2}\right)$. The plasma glucose $t_{1 / 2}$ was calculated from the slope of the least-squares analysis of plasma glucose concentrations during the linear decay phase [18]. Anesthesia was not used during the ivGTT or ipITT tests. The animals used in the ivGTT and ipITT tests were not used for further analysis. 


\section{Plasma analysis of total cholesterol, ALT and AST}

Blood samples were collected, centrifuged ( $120 \mathrm{~g}$ for $15 \mathrm{~min}$ ) and aliquots of plasma were used for the measurements of ALT and AST by the kinetic UV method using an automatic spectrophotometer and a specific commercial kit (Gold Analisa ${ }^{\circledR}$ Belo Horizonte, Minas Gerais, Brazil). Serum total cholesterol was measured by a colorimetric enzymatic method (Gold Analisa ${ }^{\circledR}$ Belo Horizonte, Minas Gerais, Brazil). Analyses were performed in triplicate [12].

\section{Pancreatic islets isolation and static insulin secretion}

Pancreatic islets were isolated by collagenase digestion and washed with Hank's solution as previously described [19]. Groups of four isolated islets were pre-incubated for $60 \mathrm{~min}$ at $37^{\circ} \mathrm{C}$ in Krebs solution containing $5.6 \mathrm{mM}$ glucose in an incubator with a mixture of $95 \% \mathrm{O}_{2}: 5 \% \mathrm{CO}_{2}$ and $\mathrm{pH} 7.4$ to stabilize insulin secretion. The Krebs solution was then replaced, and islets were incubated for 60 min under the experimental conditions with $5.6 \mathrm{mM}, 8.3 \mathrm{mM}, 11.1 \mathrm{mM}$ and $16.7 \mathrm{mM}$ glucose to assess GIIS. The insulin secretion was determined by RIA [18].

\section{Parasympathetic activity}

Following $12 \mathrm{~h}$ of fasting, animals from the four experimental groups (91 days old; $\mathrm{n}=15-18$ ) were anaesthetized (thiopental $45 \mathrm{mg} / \mathrm{kg}$ of body weight). A surgical longitudinal incision was made on the anterior cervical region. Employing a dissection microscope, the nerve bundle of the left vagus' superior branch was severed from the carotid artery close to the trachea, as previously described [6]. The neural signal output was acquired by an Insight interface (Insight ${ }^{\circledR}$, Riberão Preto SP Brazil). Nerve activity was analyzed for 5 sec to determine the number of spikes. After stabilizing of the signal for 5-10 min, 20 record frames of $15 \mathrm{sec}$ from each animal were randomly chosen for spike counting. Spikes greater than $0 \mathrm{mV}$ were assumed to be signals. The average number of spikes was used as the nerve firing rate for each rat.

\section{Sympathetic activity}

The sympathetic branch nerve (SN) from the superior cervical ganglia was dissected from another group of animals from the four experimental groups ( 91 days old; $\mathrm{n}=15$ - 18) after $12 \mathrm{~h}$ of fasting. The electrode was placed under the SN. Firing rates from the SN were obtained as described for the vagus nerve. The animals used in the parasympathetic and sympathetic activities tests were not used for further analysis.

\section{Liver histological analysis}

Several fragments from all parts of the liver from six animals per experimental group were instantly fixed in Bouin's solution, dehydrated using graded concentration solutions of ethanol $(70 \%, 80 \%, 90 \%$ and $100 \%)$, diaphanized in xylol and embedded in histologic paraffin. Transverse semi-serial sections of $5 \mu \mathrm{m}$ thickness were obtained using a Leica RM 2145 microtome (Leica Microsystems, Wechsler, Germany) with a steel knife. The histologic sections were stained with hematoxylin and eosin (H\&E). Morphology analysis was carried out using five non-consecutive digital images per animal using a high resolution camera (Q Color 3 Olympus American, Burnaby, BC, Canada) coupled to an Olympus BX 41 light microscope at $20 \times$ and $40 \times$ objectives (Olympus, Tokyo, Japan); subsequently, these images were transmitted to a computer using Q Capture Pro 5.1 and Image-Pro Plus 4.5 (Media Cybernetics, Silver Springs, MD, USA) [20, 21].

\section{Statistical analysis}

The results are expressed as the mean \pm standard error of the mean $(M \pm S E M)$. The data obtained from all experiments were compared using the one-way ANOVA. The post hoc Tukey's multiple comparison test was employed when necessary. The significance level was set at $p<0.05$. The statistical analyses were performed using GraphPad Prism ${ }^{\circledR}$ 5.0 Software, San Diego, CA, USA.

\section{Results}

Moderate exercise attenuates obesity

Body weight was significantly different between the Control-SED and HFD-SED groups from the $7^{\text {th }}$ week of HFD-treatment up to the $10^{\text {th }}$ week of treatment. In comparison with the 
Table 1. Effects of moderate exercise on food intake, biometric and plasma biochemical parameters. All values are expressed as the mean \pm SEM $(n=10-12) .{ }^{*} p<0.0001$ versus Control-SED; ${ }^{\#} p<0.05$ versus Control-SED; ${ }^{\S} p<0.01$ versus HFD-SED. One-way ANOVA, post hoc Tukey's test

\begin{tabular}{lcccc}
\hline & \multicolumn{2}{c}{ Sedentary } & \multicolumn{2}{c}{ Exercised } \\
& Control & HFD & Control & HFD \\
\hline Final body weight $(\mathrm{g})$ & $377.3 \pm 3.5$ & $440.9 \pm 7.1^{*}$ & $321.4 \pm 7.0^{\sharp}$ & $324.6 \pm 8.6^{\sharp}$ \\
Lee index & $315.2 \pm 1.7$ & $329.7 \pm 1.4^{*}$ & $316.2 \pm 1.3$ & $320.7 \pm 2.1 \S$ \\
AUC food intake (kcal) & $775.7 \pm 9.9$ & $831.3 \pm 8.9^{*}$ & $776.1 \pm 6.7$ & $837.9 \pm 8.2$ \\
Fasting glucose (mg/dL) & $90.6 \pm 2.3$ & $99.9 \pm 2.9$ & $95.4 \pm 3.6$ & $92.9 \pm 6.4$ \\
Fasting insulin (ng/mL) & $1.6 \pm 0.3$ & $3.6 \pm 0.8^{*}$ & $1.0 \pm 0.2$ & $1.1 \pm 0.2 \S$ \\
HOMA & $5.7 \pm 1.0$ & $10.9 \pm 1.7^{*}$ & $4.2 \pm 0.9$ & $5.4 \pm 1.3 \S$ \\
Kitt (\%/min) & $2.7 \pm 0.2$ & $1.2 \pm 0.2^{*}$ & $3.6 \pm 0.2^{\sharp}$ & $3.9 \pm 0.3 \S$ \\
Total cholesterol (mg/dL) & $76.8 \pm 1.8$ & $108.2 \pm 4.1^{*}$ & $71.9 \pm 2.1$ & $80.3 \pm 5.5^{\S}$ \\
ALT (U/L) & $37.2 \pm 3.5$ & $54.1 \pm 2.1^{*}$ & $34.4 \pm 2.3$ & $36.0 \pm 3.4 \S$ \\
AST (U/L) & $24.5 \pm 1.4$ & $44.3 \pm 3.5^{*}$ & $26.1 \pm 1.9$ & $25.8 \pm 4.5 \S$ \\
\hline
\end{tabular}

Fig. 2. Effect of moderate exercise and/or HFD on fat tissue accumulation in rats. (A) Periepididymal, (B) retroperitoneal (C), inguinal (D) and mesenteric fat pad. All values are expressed as the mean \pm $\operatorname{SEM}(n=10-12)$. ${ }^{*} p<0.0001$ versus Control-SED; ${ }^{\#} p<0.0001$ versus Control-SED; ${ }^{\S} p<0.0001$ versus HFD-SED. One-way ANOVA, post hoc Tukey's test.

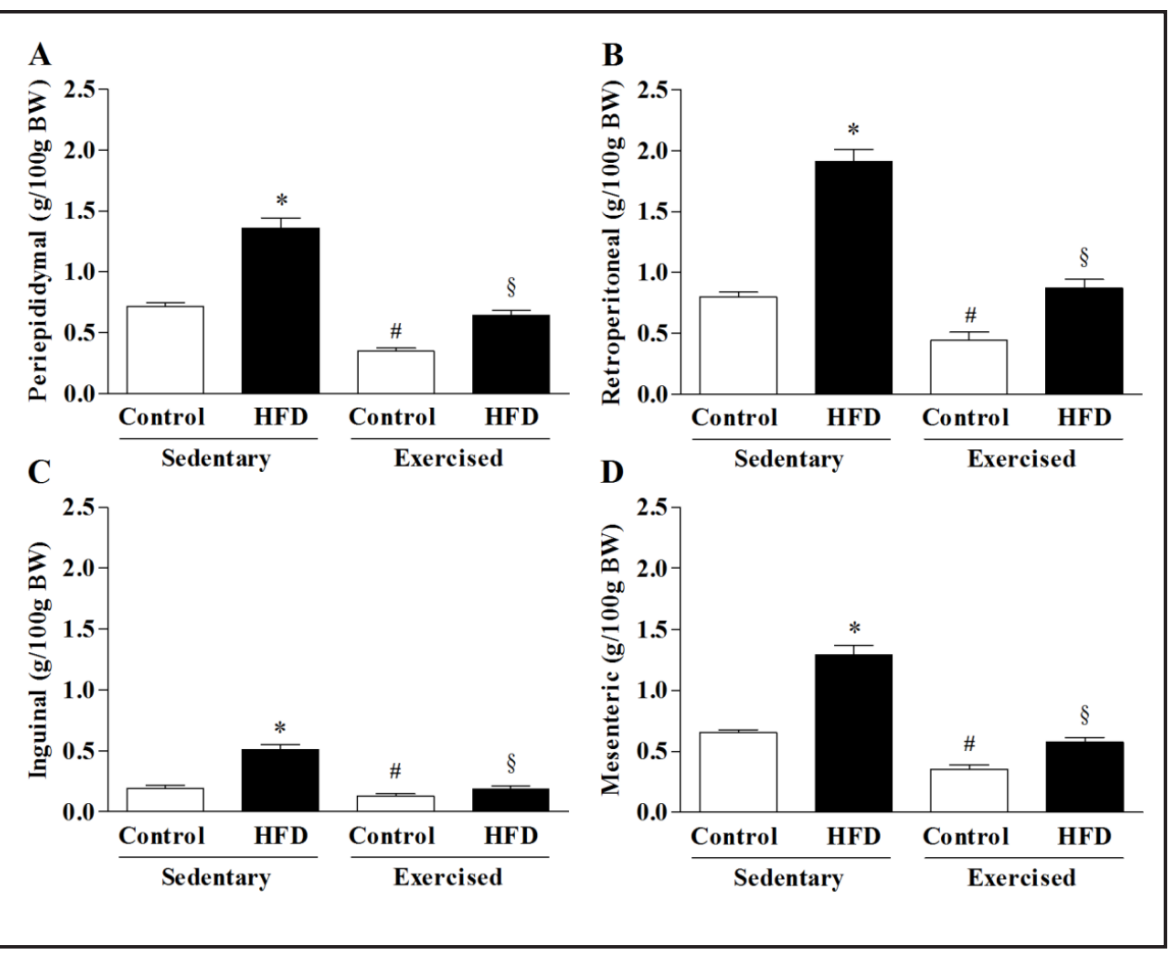

Control-SED group, the body weight of the HFD-SED group increased by $8 \%$ in the $7^{\text {th }}$ week $(307.0 \pm 4.7 \mathrm{~g}$ vs. $331.4 \pm 5.2 \mathrm{~g} ; p<0.05)$ and $17 \%$ in the $10^{\text {th }}$ week $(p<0.0001$; Table 1$)$. HFDSED animals showed an increase of $5 \%$ in the Lee index when compared to the control-SED group $(p<0.0001$; Table 1$)$. Weekly food intake (kcal) became significantly different between the Control-SED and the HFD-SED groups at the $7^{\text {th }}$ week (increased by $8 \% ; 111.7 \pm 4.0 \mathrm{kcal}$ vs. $120.8 \pm 3.9 \mathrm{kcal} ; p<0.05$ ) of HFD-treatment and continued until the $10^{\text {th }}$ week (increased by $16 \% ; 109.5 \pm 3.1 \mathrm{kcal}$ vs. $126.8 \pm 4.9 \mathrm{kcal} ; p<0.05$ ). This result is clearly demonstrated by the area under curve (AUC) of food intake during the entire 10 -week treatment period, from 21 until 91 days-old ( $p<0.05$; Table 1). Meanwhile, the food intake (grams) was decreased by $20 \%(178.9 \pm 5.1 \mathrm{~g}$ vs. $141.9 \pm 1.5 \mathrm{~g} ; p<0.05)$ in HFD-SED group in relation to ControlSED animals, and exercise did not modify this parameter. The HFD-SED group showed an increase of 90\% in the EPI (Fig. 2A), 140\% in the RP (Fig. 2B), 172\% in the ING (Fig. 2C) and $97 \%$ in the MES (Fig. 2D) fat pads in relation to the Control-SED group $(p<0.0001)$.

The treadmill exercise protocol was able to reduce the body weight (Control-EXE by $15 \%, p<0.05$ and HFD-EXE by $27 \%, p<0.0001$ ) compared to sedentary animals on similar diets (Table 1). When compared to the HFD-SED group, this protocol also resulted in a $3 \%$ 
Fig. 3. Moderate exercise reduces plasmatic levels of glucose and insulin during ivGTT and improves insulin secretion by pancreatic islets in HFD rats. (A) glycemia curves, (B) AUC of glycemia over 45 min, (C) insulinemia curves, (D) AUC of insulinemia over 45 min. (E) Insulin secretion from pancreatic islets stimulated by various glucose concentrations. All values are expressed as the mean $\pm \operatorname{SEM}(n=10$ 12). ${ }^{*} p<0.01$ versus Control-SED; ${ }^{\S} p<0.01$ versus HFD-SED. One-way ANOVA, post hoc Tukey's test.
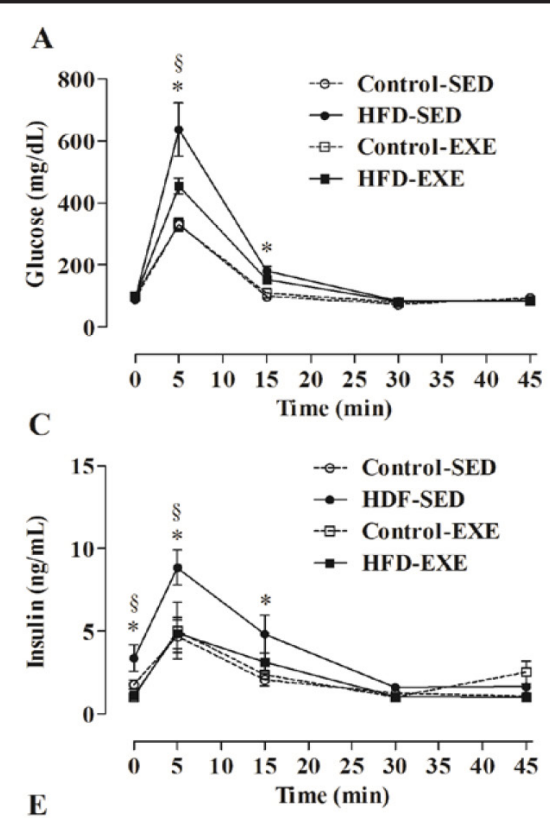

\section{B}

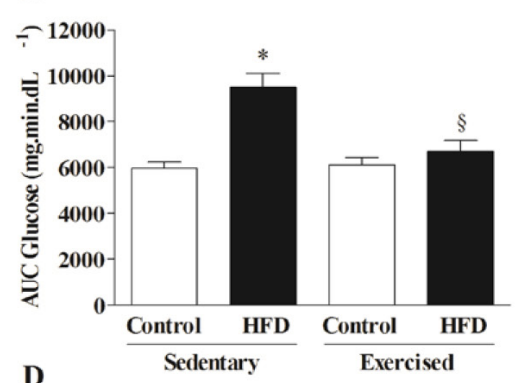

D
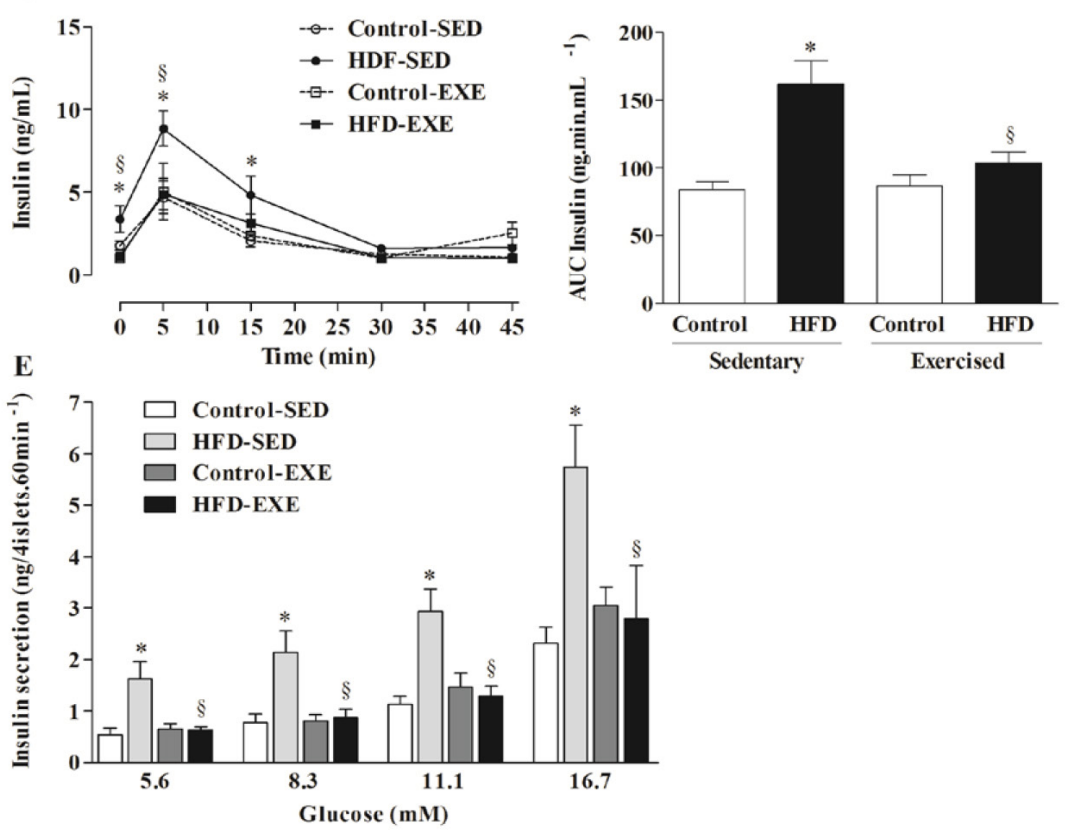

decrease in the Lee index of the HFD-EXE animals ( $p<0.001$; Table 1$)$. However, no differences were observed in the food intake between the exercised groups and the sedentary groups (Table 1). Both Control-EXE and HFD-EXE animals showed lower fat tissue accumulation (EPI 51\% and 53\%, Fig. 2A; RP 44\% and 54\%, Fig. 2B; ING 31\% and 64\%, Fig. 2C and MES fat pad $46 \%$ and 55\%, Fig. 2D, respectively) compared to Control-SED and HFD-SED groups $(p<0.0001)$.

\section{Moderate exercise improves glycemic homeostasis}

As shown in Table 1, although there was no change in the fasting glucose level, the fasting insulin and HOMA index were $124 \%$ and $89 \%$ higher $(\mathrm{p}<0.001)$ in the HFD-SED group compared with control-SED, respectively. Treadmill exercise reduced fasting blood insulin by $69 \%$ and the HOMA index by $50 \%$ in HFD-EXE ( $<$ < 0.001) compared with HFDSED rats. These metabolic parameters did not change in the Control-EXE animals compared to the Control-SED rats (Table 1).

Insulin sensitivity $\left(\mathrm{K}_{\mathrm{itt}}\right)$ during ipITT was significantly decreased (55\%) in the HFDSED group compared to the Control-SED group ( $<<0.05$; Table 1$)$. Treadmill exercise led to higher insulin sensitivity as demonstrated by the $\mathrm{K}_{\mathrm{itt}}$ values of $225 \%(\mathrm{p}<0.0001)$ and $33 \%$ $(\mathrm{p}<0.05)$ in HFD-EXE and Control-EXE groups, respectively (Table 1).

HFD-SED rats displayed higher blood glucose at 5 and 15 min during the ivGTT test $(\mathrm{p}<$ 0.01; Fig. 3A) and a 60\% increase in AUC ( $<<0.0001$; Fig. 3B) in relation to the Control-SED group. Plasma insulin levels were increased at time 0 (zero), 5 and $15 \mathrm{~min}(\mathrm{p}<0.05$; Fig. 3C) during the ivGTT, and the AUC of this parameter was one-fold higher in the HFD-SED group 
Fig. 4. Moderate exercise restores ANS activity imbalanced by the HFD in rats. (A) vagus nerve electrical activity. (B) sympathetic branch nerve activity from the superior cervical ganglia. All values are expressed as the mean \pm SEM ( $n=15$ - 18). ${ }^{*} p<0.0001$ versus Control-SED; ${ }^{\#} p<0.0001$ versus Control-EXE; ${ }^{\S} p<0.0001$ versus HFD-SED. One-way ANOVA, post hoc Tukey's test.

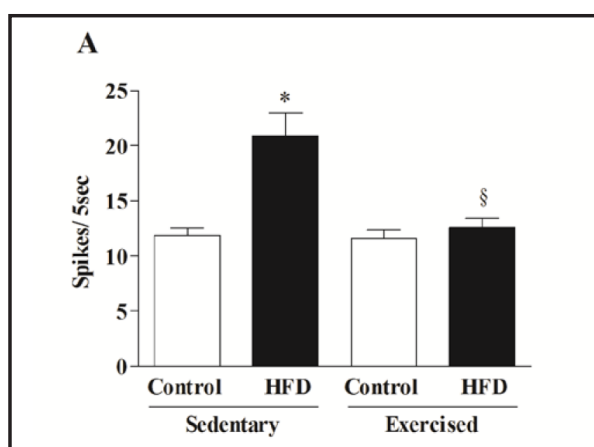

B
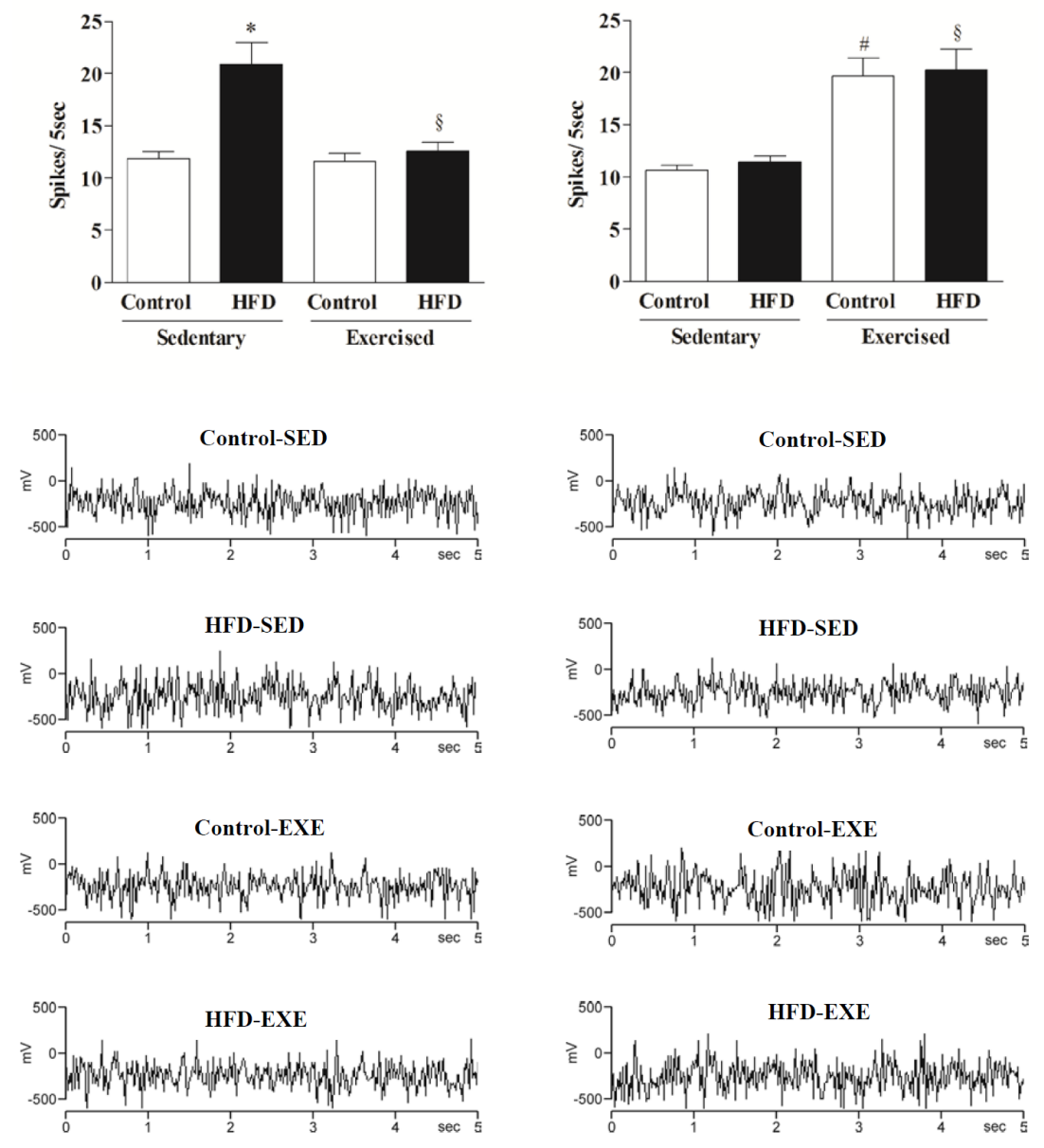

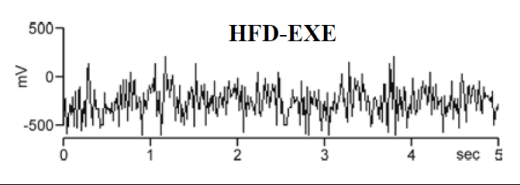

compared with the Control-SED group ( $p<0.0001$; Fig. 3D). Treadmill exercise reduced blood glucose and insulin during the ivGTT test in HFD-EXE rats. The HFD-EXE group had lower plasma glucose and insulin levels than the HFD-SED group (a decrease of 39 and 36\%, respectively, p < 0.0001; Fig. 3). No differences were observed between the Control-EXE and Control-SED groups (Fig. 3).

Effects of HFD and moderate exercise on plasma levels of total cholesterol, ALT and AST

The HFD significantly increased the plasma levels of total cholesterol (41\%), ALT (45\%) and AST (81\%) in the HFD-SED group compared to the Control-SED group ( $<<0.01)$. Treadmill exercise improved these parameters in the HFD-EXE group in relation to the HFD-SED group $(\mathrm{p}<0.01)$. No differences were observed between the Control-EXE and Control-SED groups (Table 1).

\section{Effect of HFD and moderate exercise on GIIS}

Fig. 3E shows the insulinotropic effect of glucose on isolated islets. Islets isolated from HFD-SED rats secreted more insulin than islets isolated from all of the other groups at every glucose concentration $(p<0.001)$. Exercise decreased glucose-induced insulin secretion by islets from HFD-EXE animals compared with islets from the HFD-SED animals $(\mathrm{p}<0.001)$. Insulin secretion in the Control-EXE animals was not different from the control-SED animals.

Moderate exercise improves ANS activity imbalanced by the HFD

Fig. $4 \mathrm{~A}$ shows that the vagus nerve firing rates increased $77 \%$ in the HFD-SED group when compared to the control-SED animals $(\mathrm{p}<0.0001)$. Treadmill exercise did not cause 
Fig. 5. Moderate exercise attenuates liver steatosis in HFD fed rats. Photomicrographs of livers (H\&E stain; 20× objective magnification; bars $=100 \mu \mathrm{m}$; $n=6$ ) from the Control-SED group show a typical liver (upper left panel). HFD-SED group livers show macro- and micro-vesicular steatosis (upper right panel, arrows). Moderate exercise attenuates macroand micro-vesicular steatosis in the HFD-EXE group (lower right panel). Moderate exercise training did not change the liver morphology in control rats (lower left panel) compared with the Control-SED group. $\mathrm{CV}=$ central vein.

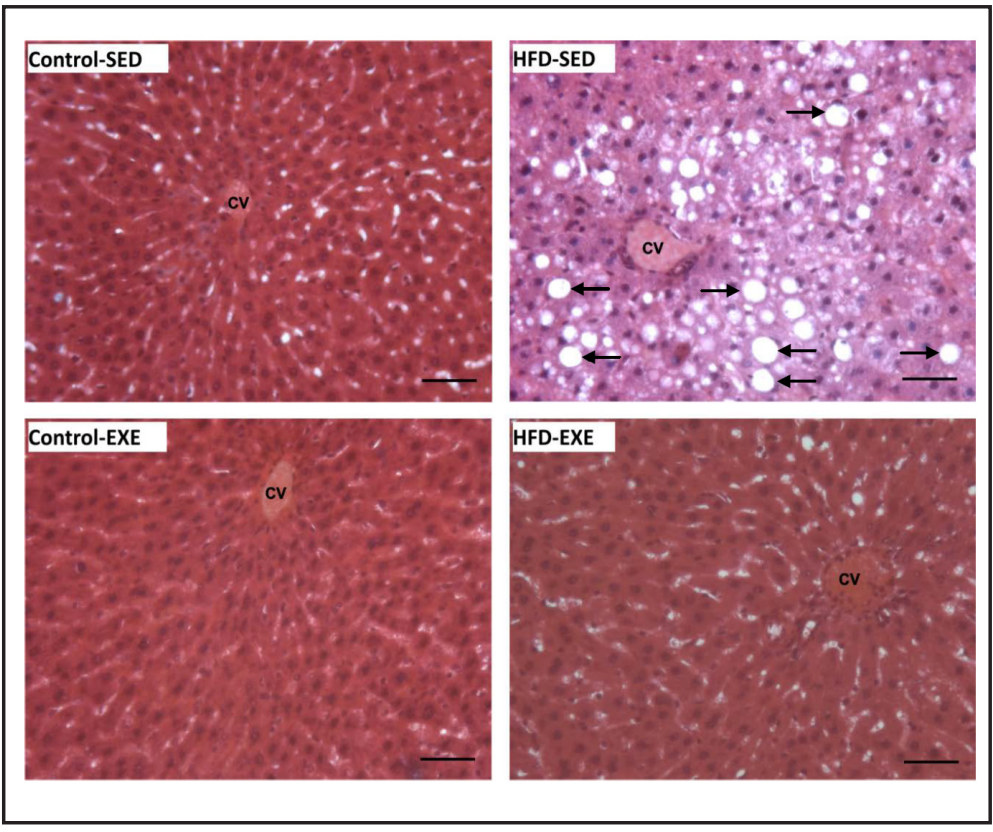

any significant modification of the number of vagus nerve spikes in the Control-EXE group. However, a $40 \%$ decrease in vagus nerve electrical activity was observed in the HFD-EXE group compared with the HFD-SED group ( $<<0.0001)$.

Fig. 4B shows that treadmill exercise increased the triggering rate of $\mathrm{SN}$ by two-fold in the Control-EXE and HFD-EXE rats $(p<0.0001)$. No change was observed in the number of SN spikes in HFD-SED rats compared with Control-SED rats.

\section{Moderate exercise attenuates liver steatosis in high-fat diet fed rats}

Photomicrographs of liver samples stained with H\&E are shown in Fig. 5. Qualitative analysis of the liver samples showed morphological changes in the livers from the HFD-SED group consisted of micro-vesicular or macro-vesicular steatosis. Alternatively, moderate exercise attenuated these morphological changes in the livers of HFD-EXE rats compared with HFD-SED rats. There were no differences between the Control-SED and the Control-EXE groups.

\section{Discussion}

For the first time, this study shows that an exercise training program, of moderate intensity and low frequency, was able to prevent some metabolic syndrome hallmarks, such as glucose intolerance, hyperinsulinemia, high fat tissue accumulation and high lipid deposits in liver. Likewise, insulin over-secretion in response to increasing glucose concentrations, displayed by isolated islets from HFD-obese rats, was also improved by this exercise training program. All benefits of this specific physical exercise program were associated with a decrease of PNS activity and an increase of SNS activity.

Our data corroborate the previous studies reporting that HFD-treated animals show higher caloric intake than animals fed a standard diet [22-24]. Moderate exercise did not modify food intake; this result agrees with research showing that no difference in food consumption was reported in trained animals compared with sedentary animals [25]. Previously, we demonstrated that MSG-obese or lean mice that underwent free swimming exercise, which was training with low intensity and low frequency, had no difference in food consumption compared with a respective sedentary group [6]. 
According to Patterson et al. non-exercised HFD-fed animals developed obesity and significant increases in fat tissue; this is also shown in the current study, where the fat mass increase occurred in the four specific fat storing regions, EPI, RP, ING and MES, proportionally [26]. The exercise program used in the current work was effective at preventing a body weight increase in control and HFD groups in comparison to their respective sedentary groups throughout the experimental period. Levin and Dunn-Meynell also reported that obese rats (HFD-treated) had a reduced body weight gain when they underwent voluntary training [27].

Our data show that high fat accumulation caused by HFD-treatment was inhibited by moderate exercise. Because this effect has been reported in controls animals, it may suggest that the physical exercise was of mild, or moderate, intensity and metabolically aerobic. This may be because the treadmill exercise protocol used in the present work consumes a significant part of the fat tissue as energy substrate. A similar effect has also been recorded in other studies [26, 28]. Veras-Silva et al. showed that a treadmill running speed of 16-20 $\mathrm{m} \times \mathrm{min}^{-1}$ corresponds to $55 \%$ of $\mathrm{VO}_{2 \max }$ in rats [14]. Physical activity stimulates the release of catecholamines in the sympathetic terminals and through the chromaffin cells of the adrenal medulla glands. This process then triggers lipolysis and lipids of the fat tissues, which are mobilized to be employed as energy substrate $[29,30]$. As previously published, our group reported that increases in the total catecholamine content were more prominent in MSGobese mice that began the exercise training in early developmental stages of life, even if these mice stopped the training close to the end of the adolescence phase. On the other hand, mice that began to swim after the adolescent period did not present improvement on the sympathoadrenal axis [30]. In the present work, we show that HFD-treated rats that run throughout HFD treatment, including the adolescent phase, displayed resistance against HFD-induced metabolic disturbance as well as beta cell malfunction. Our data are suggestive that moderate intensity physical training performed during this developmental plasticity phase might have modulated the ANS function, thereby blocking the HFD-induced metabolic malfunctions. Exercise training acts as one important modulator of ANS activity by controlling metabolism through the connections between the brain and peripheral tissues that are mainly under the action of hypothalamic-pituitary-adrenal (HPA) axis [31].

Recently, our group showed that HFD introduced during adolescence causes more drastic consequences in metabolism than when HFD was administered in adult rats [13]. As with gestation and/or lactation, adolescence is a very sensitive phase in brain development; and any injury during the peripubertal period might cause permanent changes in metabolism when the individual reaches adult life [32]. It has been shown that the earlier the exercise program is started, the more evident the inhibition of obesity onset will be [26,30].

When insulin is bound by its specific receptors, it initiates a series of phosphorylation reactions in cytosolic proteins that culminates in the translocation of the GLUT-4 vesicles to the plasma membrane. This pathway is used by glucose to enter the cells. Rodrigues et al. showed that obese animals given early over-nutrition display impaired insulin signaling with low IRS-1 and PI3K levels and, consequently, a decrease in insulin sensitivity [33]; these results confirm our findings from the ivGTT and ipITT tests. We showed that HFD-SED rats were resistant to insulin when compared to Control-SED animals, as has been observed in other studies $[8,13]$. The development of insulin resistance was inhibited by moderate exercise in the HFD-EXE group in contrast to the respective sedentary group (HFD-SED). HFD-EXE animals were normo-glycemic and secreted less insulin than the HFD-SED group. These findings suggest an improvement in insulin sensitivity caused by the exercise protocol used in this study. A low HOMA index and high $\mathrm{K}_{\mathrm{itt}}$ rates also confirm that trained HFD-fed rats did not show peripheral resistance to insulin, even though the exercises themselves triggered a significant increase in GLUT-4 expression and translocation into the skeletal muscular tissue [34,35]. Other more sensitive methods such as euglycemic clamping could be useful. In addition, our results showed that moderate exercise increased insulin sensitivity and reduced insulin secretion in the HFD-treated group. This was determined by the response of isolated pancreatic islets to basal and stimulatory glucose concentrations 
and is similar to other studies where swimming or endurance training was used $[7,18]$.

One of the major findings of this study is that moderate exercise improves pancreatic islet function disrupted by HFD treatment. We show in the current work that HFD consumption increases the release of insulin by glucose-stimulated islets. We may attribute an increase in the insulinotropic effect of glucose to an adaptation to avoid peripheral insulin resistance, which has been reported in HFD-fed animals [36]. This type of adaptation has also been seen in other models of obesity and in humans $[19,37,38]$. Decreases in peripheral insulin resistance and in the insulinotropic effect of glucose in HFD-EXE animals may confirm the previous hypothesis. However, insulin secretion decreases during physical activity and is coupled to an increase in glucagon and epinephrine secretion to promote hepatic gluconeogenesis for the maintenance of blood glucose levels [39].

For the first time, the current study shows imbalanced ANS activity, characterized by an increased firing rate from the vagus nerve caused by HFD-induced obesity. These data support the autonomic hypothesis of obesity [40]. An autonomic imbalance, revealed by electrical nerve activity in HFD-SED obese-rats, might have a strong relationship with the glycemic homeostasis rupture and high capacity of fat accumulation in tissues from obese animals. Many models of obesity, including the HFD-SED group of this study, show insulin over-secretion, which has a close relationship with high vagus activity $[6,41]$. This is because blood insulin quantity is also modulated by vagal activity in pancreatic islets. The current exercise program was able to improve autonomic activity in the HFD-EXE group; PNS, represented by vagus nerve activity, decreases, whereas SNS, represented by SN activity, increases. High PNS activity stimulates anabolic pathways such as lipogenesis, reported in obesity onset, while high SNS activity stimulates catabolic metabolism such as lipolysis. SNS stimulation has been frequently associated with exercise [6, 42, 43].

Liver steatosis is considered the hepatic manifestation of metabolic syndrome and is highly associated with increases in body mass or obesity, insulin resistance, hypertriglyceridemia, high total cholesterol, elevation of the plasma liver enzymes ALT and AST and adipokines such as leptin and adiponectin. Moreover, an HFD administration causes alterations in these parameters $[12,21]$. In the present study, the HFD-SED group developed both hepatic micro- and macro-steatosis and a significant elevation of the plasma liver enzymes ALT and AST and total cholesterol. There are multiple mechanisms for the development of liver steatosis. We suggest that our findings demonstrate a relationship between fat mass gain, insulin resistance and liver steatosis. In addition, other investigations have reported that leptin resistance and hypoadiponectinemia may also contribute to liver steatosis [21]. However, the present study shows that moderate exercise improves the plasmatic levels of ALT, AST and total cholesterol and attenuates liver steatosis. Thereby, our findings indicate that moderate-intensity and low-frequency treadmill exercise has positive effects on liver fat oxidation and significantly reduces liver steatosis via a mechanism that is similar to that caused by swimming training [12].

\section{Conclusions}

We conclude that moderate intensity and low frequency exercise training is able to attenuate obesity onset, liver steatosis and to improve insulin resistance. These effects may be closely associated with the improvement of pancreatic islet function and ANS activity that were observed in obese rats due to the consumption of a HFD. It is important to keep in mind that further studies are needed to elucidate the mechanisms by which physical exercise affects insulin sensitivity, insulin release by beta-cell, liver fat oxidation and ANS.

\section{Conflicts of Interest}

The authors declare that there are no conflicts of interest that could damage the impartiality of this scientific work. 
Gomes et al.: Moderate Exercise Restores Beta-Cell Function

\section{Acknowledgements}

This research was supported by the Brazilian Research Agencies: Conselho Nacional de Desenvolvimento Científico e Tecnológico - CNPq; Coordenação de Aperfeiçoamento de Pessoal de Nível Superior - CAPES.

\section{References}

1 Martinez JA: Body-weight regulation: Causes of obesity. Proc Nutr Soc 2000;59:337-345.

2 Moraes JC, Coope A, Morari J, Cintra DE, Roman EA, Pauli JR, Romanatto T, Carvalheira JB, Oliveira AL, Saad MJ, Velloso LA: High-fat diet induces apoptosis of hypothalamic neurons. PLoS One 2009;4:e5045.

3 Mitrani P, Srinivasan M, Dodds C, Patel MS: Autonomic involvement in the permanent metabolic programming of hyperinsulinemia in the high-carbohydrate rat model. Am J Physiol Endocrinol Metab 2007;292:E1364-1377.

4 Lakka TA, Laaksonen DE, Lakka HM, Mannikko N, Niskanen LK, Rauramaa R, Salonen JT: Sedentary lifestyle, poor cardiorespiratory fitness, and the metabolic syndrome. Med Sci Sports Exerc 2003;35:12791286.

5 Rennie KL, McCarthy N, Yazdgerdi S, Marmot M, Brunner E: Association of the metabolic syndrome with both vigorous and moderate physical activity. Int J Epidemiol 2003;32:600-606.

6 Scomparin DX, Gomes RM, Grassiolli S, Rinaldi W, Martins AG, de Oliveira JC, Gravena C, de Freitas Mathias PC: Autonomic activity and glycemic homeostasis are maintained by precocious and low intensity training exercises in msg-programmed obese mice. Endocrine 2009;36:510-517.

7 de Carvalho Leite N, Ferreira TR, Rickli S, Borck PC, de Freitas Mathias PC, de Oliveira Emilio HR, Grassiolli S: Glycolytic and mitochondrial metabolism in pancreatic islets from msg-treated obese rats subjected to swimming training. Cell Physiol Biochem 2013;31:242-256.

-8 Lalli CA, Pauli JR, Prada PO, Cintra DE, Ropelle ER, Velloso LA, Saad MJ: Statin modulates insulin signaling and insulin resistance in liver and muscle of rats fed a high-fat diet. Metabolism 2008;57:57-65.

-9 De Souza CT, Araujo EP, Bordin S, Ashimine R, Zollner RL, Boschero AC, Saad MJ, Velloso LA: Consumption of a fat-rich diet activates a proinflammatory response and induces insulin resistance in the hypothalamus. Endocrinology 2005;146:4192-4199.

10 Halade GV, Rahman MM, Williams PJ, Fernandes G: High fat diet-induced animal model of age-associated obesity and osteoporosis. J Nutr Biochem 2010;21:1162-1169.

11 Ropelle ER, Pauli JR, Prada PO, de Souza CT, Picardi PK, Faria MC, Cintra DE, Fernandes MF, Flores MB, Velloso LA, Saad MJ, Carvalheira JB: Reversal of diet-induced insulin resistance with a single bout of exercise in the rat: The role of ptp1b and irs-1 serine phosphorylation. J Physiol 2006;577:997-1007.

12 Schultz A, Mendonca LS, Aguila MB, Mandarim-de-Lacerda CA: Swimming training beneficial effects in a mice model of nonalcoholic fatty liver disease. Exp Toxicol Pathol 2012;64:273-282.

13 Barella LF, de Oliveira JC, Branco RC, Camargo RL, Gomes RM, Mendes FC, Miranda RA, Gravena C, Torrezan R, Grassiolli S, de Freitas Mathias PC: Early exposure to a high-fat diet has more drastic consequences on metabolism compared with exposure during adulthood in rats. Horm Metab Res 2012;44:458-464.

14 Veras-Silva AS, Mattos KC, Gava NS, Brum PC, Negrao CE, Krieger EM: Low-intensity exercise training decreases cardiac output and hypertension in spontaneously hypertensive rats. Am J Physiol 1997;273:H2627-2631.

15 Bernardis LL, Patterson BD: Correlation between 'lee index' and carcass fat content in weanling and adult female rats with hypothalamic lesions. J Endocrinol 1968;40:527-528.

16 Milanski M, Arruda AP, Coope A, Ignacio-Souza LM, Nunez CE, Roman EA, Romanatto T, Pascoal LB, Caricilli AM, Torsoni MA, Prada PO, Saad MJ, Velloso LA: Inhibition of hypothalamic inflammation reverses dietinduced insulin resistance in the liver. Diabetes 2012;61:1455-1462.

17 Pacini G, Mari A: Methods for clinical assessment of insulin sensitivity and beta-cell function. Best Pract Res Clin Endocrinol Metab 2003;17:305-322.

-18 Calegari VC, Zoppi CC, Rezende LF, Silveira LR, Carneiro EM, Boschero AC: Endurance training activates amp-activated protein kinase, increases expression of uncoupling protein 2 and reduces insulin secretion from rat pancreatic islets. J Endocrinol 2011;208:257-264.

19 Grassiolli S, Bonfleur ML, Scomparin DX, de Freitas Mathias PC: Pancreatic islets from hypothalamic obese rats maintain k+atp channel-dependent but not -independent pathways on glucose-induced insulin release process. Endocrine 2006;30:191-196. 
20 Scoaris CR, Rizo GV, Roldi LP, de Moraes SM, de Proenca AR, Peralta RM, Natali MR: Effects of cafeteria diet on the jejunum in sedentary and physically trained rats. Nutrition 2010;26:312-320.

-21 Trevenzoli IH, Rodrigues AL, Oliveira E, Thole AA, Carvalho L, Figueiredo MS, Toste FP, Neto JF, Passos MC, Lisboa PC, Moura EG: Leptin treatment during lactation programs leptin synthesis, intermediate metabolism, and liver microsteatosis in adult rats. Horm Metab Res 2010;42:483-490.

-22 Velkoska E, Cole TJ, Morris MJ: Early dietary intervention: Long-term effects on blood pressure, brain neuropeptide y, and adiposity markers. Am J Physiol Endocrinol Metab 2005;288:E1236-1243.

23 Hansen MJ, Jovanovska V, Morris MJ: Adaptive responses in hypothalamic neuropeptide y in the face of prolonged high-fat feeding in the rat. J Neurochem 2004;88:909-916.

-24 Shiraev T, Chen H, Morris MJ: Differential effects of restricted versus unlimited high-fat feeding in rats on fat mass, plasma hormones and brain appetite regulators. J Neuroendocrinol 2009;21:602-609.

25 Gobatto CA, Mello MA, Souza CT, Ribeiro IA: The monosodium glutamate (msg) obese rat as a model for the study of exercise in obesity. Res Commun Mol Pathol Pharmacol 2002;111:89-101.

26 Patterson CM, Dunn-Meynell AA, Levin BE: Three weeks of early-onset exercise prolongs obesity resistance in dio rats after exercise cessation. Am J Physiol Regul Integr Comp Physiol 2008;294:R290-301.

27 Levin BE, Dunn-Meynell AA: Chronic exercise lowers the defended body weight gain and adiposity in dietinduced obese rats. Am J Physiol Regul Integr Comp Physiol 2004;286:R771-778.

-28 Scomparin DX, Grassiolli S, Gomes RM, Torrezan R, de Oliveira JC, Gravena C, Pera CC, Mathias PC: Lowintensity swimming training after weaning improves glucose and lipid homeostasis in msg hypothalamic obese mice. Endocr Res 2011;36:83-90.

-29 Scheurink AJ, Steffens AB, Dreteler GH, Benthem L, Bruntink R: Experience affects exercise-induced changes in catecholamines, glucose, and ffa. Am J Physiol 1989;256:R169-173.

-30 Scomparin DX, Grassiolli S, Marcal AC, Gravena C, Andreazzi AE, Mathias PC: Swim training applied at early age is critical to adrenal medulla catecholamine content and to attenuate monosodium l-glutamate-obesity onset in mice. Life Sci 2006;79:2151-2156.

- 31 Park E, Chan O, Li Q, Kiraly M, Matthews SG, Vranic M, Riddell MC: Changes in basal hypothalamo-pituitaryadrenal activity during exercise training are centrally mediated. Am J Physiol Regul Integr Comp Physiol 2005;289:R1360-1371.

-32 Tsoory MM, Guterman A, Richter-Levin G: "Juvenile stress" alters maturation-related changes in expression of the neural cell adhesion molecule 11 in the limbic system: Relevance for stress-related psychopathologies. J Neurosci Res 2010;88:369-380.

-33 Rodrigues AL, De Souza EP, Da Silva SV, Rodrigues DS, Nascimento AB, Barja-Fidalgo C, De Freitas MS: Low expression of insulin signaling molecules impairs glucose uptake in adipocytes after early overnutrition. J Endocrinol 2007;195:485-494.

-34 Zachwieja JJ, Hendry SL, Smith SR, Harris RB: Voluntary wheel running decreases adipose tissue mass and expression of leptin mrna in osborne-mendel rats. Diabetes 1997;46:1159-1166.

35 Chiu LL, Chou SW, Cho YM, Ho HY, Ivy JL, Hunt D, Wang PS, Kuo CH: Effect of prolonged intermittent hypoxia and exercise training on glucose tolerance and muscle glut4 protein expression in rats. J Biomed Sci 2004;11:838-846.

-36 Dobbins RL, Szczepaniak LS, Myhill J, Tamura Y, Uchino H, Giacca A, McGarry JD: The composition of dietary fat directly influences glucose-stimulated insulin secretion in rats. Diabetes 2002;51:1825-1833.

- 37 Andreazzi AE, Scomparin DX, Mesquita FP, Balbo SL, Gravena C, De Oliveira JC, Rinaldi W, Garcia RM, Grassiolli S, Mathias PC: Swimming exercise at weaning improves glycemic control and inhibits the onset of monosodium l-glutamate-obesity in mice. J Endocrinol 2009;201:351-359.

-38 Slentz CA, Tanner CJ, Bateman LA, Durheim MT, Huffman KM, Houmard JA, Kraus WE: Effects of exercise training intensity on pancreatic beta-cell function. Diabetes Care 2009;32:1807-1811.

- 39 Suh SH, Paik IY, Jacobs K: Regulation of blood glucose homeostasis during prolonged exercise. Mol Cells 2007;23:272-279.

40 Bray GA, Inoue S, Nishizawa Y: Hypothalamic obesity. The autonomic hypothesis and the lateral hypothalamus. Diabetologia 1981;20:S366-377.

41 Balbo SL, Grassiolli S, Ribeiro RA, Bonfleur ML, Gravena C, Brito Mdo N, Andreazzi AE, Mathias PC, Torrezan R: Fat storage is partially dependent on vagal activity and insulin secretion of hypothalamic obese rat. Endocrine 2007;31:142-148.

42 Romijn JA, Fliers E: Sympathetic and parasympathetic innervation of adipose tissue: Metabolic implications. Curr Opin Clin Nutr Metab Care 2005;8:440-444.

43 Giordano A, Song CK, Bowers RR, Ehlen JC, Frontini A, Cinti S, Bartness TJ: White adipose tissue lacks significant vagal innervation and immunohistochemical evidence of parasympathetic innervation. Am J Physiol Regul Integr Comp Physiol 2006;291:R1243-1255. 